\title{
DIFFERENTIAL ADSORPTION OF COMPLEX ORGANIC MOLECULE ISOMERS ON INTERSTELLAR ICE SURFACES
}

\author{
M. Bertin ${ }^{1}$, X. Michaut ${ }^{1}$, M. Lattelais ${ }^{2}$, H. Mokrane ${ }^{3}$, F. Pauzat ${ }^{2}$, \\ J. Pilmé ${ }^{2}$, C. Minot ${ }^{2}$, Y. Ellinger ${ }^{2}$, C. Romanzin ${ }^{1}$, P. Jeseck ${ }^{1}$, \\ J.-H. Fillion ${ }^{1}$, H. Chaabouni ${ }^{3}$, E. Congiu ${ }^{3}$, F. Dulieu ${ }^{3}$, S. Baouche ${ }^{3}$ \\ and J.-L. Lemaire ${ }^{3}$
}

\begin{abstract}
We present a combined theoretical and experimental study of the adsorption of two pairs of organic isomers, $(i)$ acetic acid AA $\left(\mathrm{CH}_{3} \mathrm{COOH}\right)$ and methyl formate $\mathrm{MF}\left(\mathrm{HCOOCH}_{3}\right)$, and $(\mathrm{ii})$ ethanol EtOH $\left(\mathrm{CH}_{3} \mathrm{CH}_{2} \mathrm{OH}\right)$ and dimethyl ether DME $\left(\mathrm{CH}_{3} \mathrm{OCH}_{3}\right)$, onto crystalline water ice surfaces at low temperatures. Both approaches show that, for each pair, the most stable isomer from a thermodynamical point of view, i.e. AA and $\mathrm{EtOH}$, is also the one which is the more tightly bound to the water ice surface compared to the less stable isomers (MF and DME). This finding, which can be explained by the ability of AA or EtOH to efficiently interact with the ice surface via hydrogen bondings, may have important consequences in an astrophysical context, since it could explain why the most stable isomer is not the most abundant observed in the interstellar gas phase.
\end{abstract}

\section{Introduction}

The colder regions of the interstellar medium (ISM) are known to show a large variety of molecular species. These molecules (except $\mathrm{H}_{2}$ ) form icy mantles, mainly composed of $\mathrm{H}_{2} \mathrm{O}$, and to a lesser extent some simple molecules like $\mathrm{CO}, \mathrm{CO}_{2}$ or $\mathrm{CH}_{3} \mathrm{OH}$, accreted at the surface of carbonaceous or siliceous dust grains. In the vicinity of thermal sources like protostars, i.e. in hot cores or hot corinos, the sublimation of these ices reveals the presence of more complex organic molecules

${ }^{1}$ UPMC, Université Paris 6, Laboratoire de Physique Moléculaire pour l'Atmosphère et l'Astrophysique (LPMAA) - CNRS UMR 7092, 75252 Paris, France

${ }^{2}$ UPMC Université Paris 6, Laboratoire de Chimie Théorique (LCT) - CNRS UMR 7616, 75252 Paris, France

${ }^{3}$ Observatoire de Paris et Université de Cergy Pontoise, LAMAp/LERMA - CNRS UMR 8112, 95000 Cergy Pontoise Cedex, France 
$(\mathrm{COMs})$ in the gas phase. It is now well accepted that gas phase chemistry alone cannot account for the diversity of COMs observed in the ISM and that solid-gas reactions, and even chemical processes involving adsorbed partners, have to be considered (e.g. Horn et al. 2004; Garrod \& Herbst 2006; Bennett \& Kaiser 2007). Knowing that the composition of molecular ices does not reflect the composition of the surrounding gas phase, we address the question whether adsorption at the surface of these ices could affect the abundances observed in the surrounding gas phase. To this end we consider whether isomers of complex organic molecules could provide useful information.

In a previous study we found that, when several isomers of same generic formula are identified, the most stable one was generally the most abundant observed in the gas phase. It was suggested that the few exceptions to what can be seen as a Minimum Energy Principle (Lattelais et al. 2009, 2010) could be rationalized either by the existence of different routes of formation with different kinetics or no intermediate in common and/or specific depletion on the grains of one isomer with respect to the others. It is the last option that we address here in a joint - theoretical/experimental - study of the adsorption of two pairs of isomers on crystalline water ice surfaces: $(i)$ acetic acid $\mathrm{AA}\left(\mathrm{CH}_{3} \mathrm{COOH}\right)$ and methyl formate $\mathrm{MF}\left(\mathrm{HCOOCH}_{3}\right)$, and $(\mathrm{ii})$ ethanol EtOH $\left(\mathrm{CH}_{3} \mathrm{CH}_{2} \mathrm{OH}\right)$ and dimethyl ether DME $\left(\mathrm{CH}_{3} \mathrm{OCH}_{3}\right)$. A focus has been made on the determination of the adsorption energy associated with each of the four above-mentioned compounds, which is directly linked to the strength of the attractive interactions between the water surface and the adsorbates.

\section{Theoretical approach}

In order to theoretically predict the value of the adsorption energies, the density functional theory (DFT) method has been employed. The $\mathrm{H}_{2} \mathrm{O}$ ice surface and bulk has been modeled by a periodic representation of the solid water in its crystalline, apolar variety of hexagonal ice, composed of bilayers of water molecules (Casassa et al. 2005). The unit cell that has been used takes into account two bilayers of water molecules, which can be extended to infinite dimensions using periodic limits conditions. Regarding the low abundances of COMs in the ISM compared to water, caution has been taken on the dimensions of the unit cell so that lateral interactions of the organic adsorbates on the surface are minimized and can be neglected. Practically, the calculations were then realized using VASP package (Kresse \& Hafner 1994), and a PW91 functionnal (Perdew et al. 1992). The details of the calculation methods are given in Lattelais et al. (2011).

For the AA/MF isomer pair, the results highlight different adsorption geometries and energies. Acetic acid is adsorbed in a bidentate way: the - $\mathrm{COOH}$ function is involved in two hydrogen bonds with the surface water molecules, resulting in a strong adsorption energy of $16.2 \mathrm{kcal} . \mathrm{mol}^{-1}$. The methyl formate molecule binds to the water surface via only one hydrogen bond between its $\mathrm{C}=\mathrm{O}$ group and a surface dangling O-H. Its adsorption energy, $\mathrm{E}_{a d s}=9.2 \mathrm{kcal}_{\mathrm{mol}}{ }^{-1}$ is found to be comparatively weaker than the one of the AA, which is consistent with the 
number and strength of predicted hydrogen bonds for each species' adsorption. Similar findings have been obtained for the $\mathrm{EtOH} / \mathrm{DME}$ isomer pair: ethanol has been found to form two hydrogen bonds with the water, each involving the hydrogen or the oxygen of the hydroxyl $-\mathrm{OH}$ group. On the contrary, the dimethyl ether can form only one hydrogen bond with the $\mathrm{H}_{2} \mathrm{O}$ surface through its $\mathrm{C}=\mathrm{O}$ group. The difference in adsorption energies $\left(\mathrm{E}_{a d s}=13.5 \mathrm{kcal} . \mathrm{mol}^{-1}\right.$ for EtOH and $\mathrm{E}_{a d s}=8.4 \mathrm{kcal}_{\mathrm{mol}}{ }^{-1}$ for DME) matches the difference between the number of hydrogen bonds between the two adsorption configurations.

\section{Experimental approach}

The adsorption of isomers on crystalline water ice has been experimentally studied in two ultrahigh vacuum $\left(P=1.10^{-10}\right.$ Torr $)$ setups: AA/MF was studied in the SPICES (Surface Processes and ICES) installation at UPMC, while the work on EtOH/DME has been addressed with the FORMOLISM setup at the "Université de Cergy-Pontoise". In both cases, the organics' adsorption energies on the crystalline water surface have been estimated using the TPD technique, where an in - situ grown crystalline water ice supporting a single monolayer of the COMs is linearly warmed from $70 \mathrm{~K}$ to $200 \mathrm{~K}$ while the desorbing species are probed by means of a quadrupole mass spectrometer. More details on this technique as well as on the ice growth can be found in Bertin et al. (2011). The obtained curves give the desorbing rates (i.e. the amount of desorbing molecules per second) as a function of sample temperature. Adsorption energies are derived by using the Polanyi-Wigner equations (Dejong \& Niemantsverdriet 1990). One can extract an estimation of these energies from the TPD curves in the case of (sub)monolayer adsorbate coverage regime (see Lattelais et al. 2011).

The TPD experiments have revealed that, in the submonolayer regime, the methyl formate monolayer on crystalline water surface desorbs between $115 \mathrm{~K}$ and $135 \mathrm{~K}$ when a heating rate of $1 \mathrm{~K} \cdot \mathrm{min}^{-1}$ is applied. The corresponding adsorption energy has been estimated to be $8.9 \pm 1.0 \mathrm{kcal}^{\mathrm{mol}}{ }^{-1}$. The case of acetic acid monolayer desorption is different. Indeed, it is not possible, in the TPD experiments, to differentiate the desorption of AA from the desorption of the ice substrate, since both are simultaneously observed. This common sublimation is a signature that the interaction energy between single AA molecules and the water surface is stronger than the cohesion energy of the water ice itself, which has been estimated at $11.5 \mathrm{kcal} \cdot \mathrm{mol}^{-1}$ (Fraser et al. 2001). The same behaviour has been observed for the EtOH/DME pair. Indeed, it has not been possible to discriminate between the desorption of the first EtOH monolayer and of the water substrate, whereas the thermal desorption of the DME monolayer is clearly seen at lower temperature, allowing estimation of its adsorption energy on water at $8.1 \pm 1.0 \mathrm{kcal}_{\mathrm{mol}}{ }^{-1}$.

\section{Discussion and conclusions}

The present study shows that both theory and TPD experiments agree in terms of the relative adsorption energies of the different species whenever measurement 
is possible: $\mathrm{E}_{a d s}\left(\mathrm{HCOOCH}_{3}\right)=9.2 \mathrm{kcal} \cdot \mathrm{mol}^{-1}$ (theory) and $8.9 \pm 1.0 \mathrm{kcal}^{-\mathrm{mol}^{-1}}$ $(\mathrm{TPD}) ; \mathrm{E}_{a d s}\left(\mathrm{CH}_{3} \mathrm{OCH}_{3}\right)=8.4 \mathrm{kcal} \cdot \mathrm{mol}^{-1}$ (theory) and $8.1 \pm 1.0 \mathrm{kcal} . \mathrm{mol}^{-1}$ (TPD). For the isomers that desorb with the ice $\mathrm{E}_{a d s}\left(\mathrm{CH}_{3} \mathrm{COOH}\right)=16.2 \mathrm{kcal}^{\mathrm{mol}} \mathrm{mol}^{-1}$ (theory), and $\mathrm{E}_{a d s}\left(\mathrm{CH}_{3} \mathrm{CH}_{2} \mathrm{OH}\right)=13.5 \mathrm{kcal} \mathrm{mol}^{-1}$ (theory), which are higher than the cohesion energy of crystalline water ice $\left(11.5 \mathrm{kcal}^{\mathrm{mol}}{ }^{-1}\right)$.

These differences in the adsorption energies for a same isomers pair are not related to the intrinsic stability of the molecules, but linked to the fact that both AA and EtOH make more and stronger hydrogen bonds with the ice surface than MF and DME. Therefore, the present findings may be generalized to other molecules presenting similar chemical nature (esters vs. carboxylic acids, ethers vs. alcohols). Such a differential adsorption behaviour could therefore play a role in the gas phase composition observed in the ISM. Indeed, a stronger interaction of the molecules with the icy mantles of the grains should result in a larger depletion in the gas phase for a given temperature. In this context, observational data are needed in addition to the laboratory studies to constrain the impact of the adsorption/desorption processes on the gas phase abundances observed in the ISM. Finally, one can notice that, in the case of AA/MF and EtOH/DME, the most tightly bond isomer is also the one which is the most stable (AA and EtOH). Interestingly, these two molecules are observed to be relatively less abundant compared to the less stable isomers MF and DME, and thus appear as exceptions to the Minimum Energy Principle (MEP) (Lattelais et al. 2009, 2010). If the adsorption energy plays a non-negligible role in determining the observed gas phase relative abundances, the differential adsorption process addressed in this study could at least partly explain these exceptions to the MEP.

\section{References}

Bennett, C.J., \& Kaiser, R.I., 2007, ApJ, 660, 1289

Bertin, M., et al., 2011, J. Phys. Chem. C, 115, 12920

Casassa, S., et al., 2005, Chem. Phys. Lett., 409, 110

Dejong, A.M., \& Niemantsverdriet, J.W., 1990, Surf. Sci., 233, 355

Fraser, H.J., Collings, M.P., McCoustra, M.R.S., \& Williams, D.A., 2001, MNRAS, 327, 1165

Garrod, R.T., \& Herbst, E., 2006, A\&A, 457, 927

Horn, A., Mollendal, H., Sekiguchi, O., et al., 2004, ApJ, 611, 605

Kresse, G., \& Hafner, J., 1994, J. Phys.-Cond. Matt., 6, 8245

Lattelais, M., Pauzat, F., Ellinger, Y., \& Ceccarelli, C., 2009, ApJ, 696, L133

Lattelais, M., Pauzat, F., Ellinger, Y., \& Ceccarelli, C., 2010, A\&A, 519, 7

Lattelais, M., et al., 2011, A\&A, 532, 6

Perdew, J.P., et al., 1992, Phys. Rev. B, 46, 6671 